\title{
Egresados: razones para estudiar la carrera profesional de Nutrición - Universidad Nacional Mayor de San Marcos, 2006
}

\author{
Margot Quintana 1,2, Rosa Bardález 2,3, Carmen Villarreal 2,4
}

Resumen

Palabras clave

\begin{abstract}
Objetivo: Identificar las razones de los egresados para estudiar la carrera profesional de Nutrición. Diseño: Estudio cualicuantitativo exploratorio. Lugar: Escuela Académico Profesional de Nutrición, Universidad Nacional Mayor de San Marcos. Participantes: Egresados de la Escuela de Nutrición. Intervenciones: Previo consentimiento informado, se aplicó dos grupos focales con 7 participantes cada uno, dos entrevistas en profundidad y un cuestionario a 34 egresados. Principales medidas de resultados: Razones para postular, continuar y concluir sus estudios de Nutrición. Resultados: Del total, 73\% eran mujeres, edad promedio 22,4 años, 30 alumnos respondieron la encuesta. Eligieron Nutrición porque alguien personalmente les informó sobre la carrera (30\%), en la academia pre-universitaria escuchó sobre ella (23\%), posibilidad de trasladarse (20\%), menor puntaje para ingresar (17\%). La continuaron porque tuvieron cursos motivadores y por su desempeño práctico en campo. Concluyeron, porque tiene diferentes campos de acción (73\%), para contribuir a mejorar la situación de salud y gustan trabajar directamente con la población. Percibían que tenían adecuada preparación académica, hubiesen preferido mayor práctica y capacitación en algunos temas, como nutrición deportiva. Esperan desempeñarse en nutrición comunitaria o clínica. Conclusiones: Los egresados ingresaron a la carrera profesional de Nutrición por influencia externa y por conveniencia para trasladarse a otra carrera, continuaron por los cursos motivadores y la concluyeron por convicción propia, ante la variedad de áreas de desempeño laboral.
\end{abstract}

Nutrición; educación nutricional; nutricionista.
Graduates: reasons to study Nutrition as a profession - Universidad Nacional Mayor de San Marcos, 2006

\section{Abstract}

Objective: To identify the reasons why graduates chose Nutrition as their professional career. Design: Quali-quantitative and exploratory research. Setting: Professional Academic School of Nutrition of San Marcos National Major University. Participants: Nutrition graduates. Interventions: After informed consents, two focus groups with 7 participants each, two in-depth interviews and a questionnaire to 34 former students were provided. Main

1 Centro de Investigación de Bioquímica y Nutrición. Facultad de Medicina, UNMSM. Lima, Perú.

2 Escuela Académico Profesional de Nutrición. Facultad de Medicina, UNMSM. Lima, Perú.

3 Departamento Académico de Medicina. Facultad de Medicina, UNMSM. Lima, Perú.

4 Instituto de BiologíaAndina. Facultad de Medicina, UNMSM. Lima, Perú. outcome measures: Reasons why they chose, continued and finished their Nutrition studies. Results: In summary 73\% of the participants were women, average age was 22,4 years, 30 students answered the questionnaire. They chose Nutrition because somebody personally told them about the career (30\%), they learned of it at the pre-college academy (23\%), possible transfer to another career (20\%), and lower score required for admission (17\%). They continued the career because the courses were interesting and they enjoyed the practical applications. They graduated from Nutrition because of many employment opportunities (73\%), for health improvement contribution, and to work directly with the population. They felt good about their academic preparation but they wanted more practice and qualification in subjects like sport nutrition. Desired work included communitarian or clinical nutrition. Conclusions: The participants chose Nutrition due to external influences and convenience to transfer to another career, continued by motivating courses and graduated in this area due to opportunities on diverse labour fields.

Key words: Nutrition; nutrition education; nutritionist. 


\section{INTRODUCCIÓN}

En algunas universidades se investiga las razones que tienen sus estudiantes para seguir una carrera profesional. En la literatura revisada, se exponen las razones que tuvieron los alumnos del área social y del área de salud. En el primer grupo, se tiene las de los estudiantes de educación $\left({ }^{1,2}\right)$ y periodismo $\left({ }^{3}\right)$. Mientras que del área de salud, la investigación corresponde principalmente a las de estudiantes y/o egresados de enfermería $\left({ }^{4,5}\right)$, odontología $\left({ }^{6}\right)$ y medicina $\left({ }^{7-9}\right)$, pero no a las de nutrición.

Las razones varían entre carreras profesionales y se encuentran la autorrealización y conocimiento, reconocimiento, seguridad, poder, deseos de ayudar a los demás, entre otras.

Un estudio realizado sobre el tema aplicó un cuestionario, basado en datos obtenidos en discusiones informales con los estudiantes $\left({ }^{2}\right)$, y otro estudio aplicó un cuestionario construido sobre una base de datos obtenidos en grupos focales y entrevistas $\left({ }^{6}\right)$. La presente investigación aplicó esta última metodología, haciéndo uso de la investigación cualitativa $\left({ }^{10,11}\right)$, que ofrece técnicas especializadas para obtener respuestas a fondo acerca de lo que las personas piensan y sienten. Este tipo de investigación es de índole interpretativa y se realiza con pequeños grupos de personas.

En un estudio sobre los estudiantes ingresantes 2003 a la Universidad Nacional Mayor de San Marcos ( $\left.{ }^{12}\right)$ se halló que postularon a ella por su prestigio (49\%) y calidad académica (31\%). San Marcos y su Escuela de Medicina tienen un prestigio que data de muchos años. La Escuela Académico Profesional de Nutrición (EAPN), con 31 años de vida Institucional, es una de las cinco Escuelas formadoras de profesionales de la salud de la Facultad de Medicina de la UNMSM.
Reconocer cuáles fueron las razones del egresado para postular, continuar y concluir la carrera profesional de Nutrición servirán como referencia para la evaluación curricular y toma de decisiones para el mejoramiento continuo de la calidad del servicio que presta la institución. Además, el conocer dichas razones facilitará que la función tutorial apoye eficientemente el proceso educativo.

El objetivo del presente trabajo fue identificar las razones por las que el egresado de la EAPN estudió la carrera profesional de Nutrición.

\section{MATERIALES Y MÉTODOS}

Estudio cualicuantitativo, exploratorio. La población la constituyeron los egresados de la EAPN de la Facultad de Medicina de la Universidad de San Marcos, y la muestra los 34 estudiantes ingresantes 2001 y que egresaron el 2005. La toma de datos se efectuó a los cuatro meses de haber finalizado sus estudios. El único criterio de inclusión fue que aceptaran participar en la investigación; para ello, se contó con un formato de consentimiento informado.

Se obtuvo información de los aspectos generales del participante, como edad, sexo, estado civil, colegio de procedencia y actividad de los padres obtenidos de su Ficha de datos, en los archivos de la EAPN. Por muestreo intencional se convocó a 18 egresados para participar en los grupos focales y a dos, uno por sexo, en las entrevistas a profundidad.

Los dos grupos focales fueron realizados en un ambiente acondicionado para tal fin, en la EAPN. No hubo estímulo externo o interno que distrajera la atención, fueron grabados y filmados. Se contó con una guía de 17 preguntas, donde se indagó qué fue lo que les motivó a postular a Nutrición, por qué continuaron sus estudios, cómo influyó su preparación académica para 
ello, qué recomendarían para que sus futuros compañeros se vean motivados a terminar la carrera, por qué decidieron terminar la carrera, cuáles eran sus expectativas profesionales y académicas, en qué área les agradaría desempeñarse, si recomendarían a otras personas estudiar Nutrición y si ya habían tenido alguna experiencia laboral en la carrera. Posteriormente, las grabaciones fueron transcritas.

Las dos entrevistas a profundidad tuvieron las mismas consideraciones anteriores, excepto la filmación.

A partir de las respuestas obtenidas con las dos técnicas anteriores, se diseñó un cuestionario con 19 ítems, donde se indagaba qué querían estudiar cuando estaban en el colegio, por qué postularon a San Marcos, cuántas veces postularon a la universidad antes de ingresar a Nutrición, si creían que habían decidido bien al quedarse estudiando Nutrición, qué expectativas tenían de la carrera, si ya habían tenido alguna experiencia laboral, si recomendarían estudiar nutrición a sus amigos o familiares y si había nutricionistas a los que quisieran imitar, superar o que los hayan estimulado a estudiar la carrera. Este cuestionario fue aplicado a los 34 egresados en dos formas alternativas: llenado directo o correo electrónico.

Los datos cualitativos fueron analizados según su contenido. Las transcripciones fueron agrupadas por temas, luego codificadas y se desarrolló categorías principales por temas. Se usó la estadística descriptiva con el programa Excel para los datos cuantitativos.

\section{RESULTADOS}

Se obtuvo información general de 34 recién egresados (Tabla 1).

Se formó dos grupos focales, con 7 participantes en cada uno, y dos entrevistas a
Tabla 1. Características generales de los egresados de la EAP Nutrición - UNMSM.

\begin{tabular}{lc}
\hline Edad (promedio en años) & 22,6 \\
Estado civil: soltera (o) (\%) & 100,0 \\
Sexo: (\%) & \\
$\quad$ Femenino & 73,3 \\
$\quad$ Masculino & 26,7 \\
Vive con ambos padres (\%) & 89,0 \\
Colegio de procedencia (\%) & \\
$\quad$ Privado & 60,0 \\
$\quad$ Estatal & 40,0 \\
Ocupación del padre (\%) & \\
$\quad$ Profesional independiente & 46,0 \\
$\quad$ Independiente no profesional & 40,0 \\
$\quad$ Jubilado & 14,0 \\
Ocupación de la madre (\%) & \\
$\quad$ Ama de casa & 65,0 \\
$\quad$ Profesional dependiente & 19,0 \\
Profesional independiente & 16,0 \\
\hline
\end{tabular}

profundidad. Respondieron el cuestionario 30 participantes.

Díez egresados informaron que, cuando estaban en el colegio, habían pensado en estudiar medicina, 5 odontología, 3 nutrición, 3 administración y el resto en otras carreras.

Cinco estudiantes que postularon a una universidad por primera vez, y que fue a Nutrición, tenían la intención de estudiar la carrera.

Postularon a la UNMSM, por su buena reputación y calidad académica. Solo tres de los seis que ingresaron con la intención de trasladarse a otra carrera lo intentaron.

Dentro de las razones para ingresar a Nutrición, se encontró que alguien (nutricionista, representantes de universidades que enseñan Nutrición, familiar) le informó de la carrera (30\%), en la academia preuniversitaria escuchó sobre la carrera $(23 \%)$, la posibilidad de trasladarse posteriormente $(20 \%)$ y menor puntaje para ingresar a la Facultad de Medicina (17\%). 
Tabla 2. Egresados: razones para estudiar la carrera profesional de Nutrición en la UNMSM.

Para postular a la carrera de Nutrición

Influencia externa

Conveniencia para traslado a otra carrera profesional

Menor puntaje para ingreso

Para continuar estudiando la carrera

Cursos motivadores

Expectativas satisfechas de desempeño en campo

Para concluir la carrera

Diversidad en el campo laboral

Participación en la mejora de la situación de salud

Trabajo directo con la población
“Conocí a una persona que era nutricionista, le iba bien, me habló y me gustó. Yo no sabía que existía la carrera en el país” (mujer 22 años).

“En la Pre (academia pre-universitaria) escuché sobre Nutrición” (mujer, 23 años).

“Tenía pensando realizar mi traslado interno una vez ingresado" (mujer, 23 años).

“Porque el puntaje (para ingresar a la universidad) era más bajo (risas)” (varón, 22 años).

“Me gustó desde el curso de ICAN (Introducción a la Ciencia de la Alimentación y Nutrición”) (varón, 21 años)

“En el segundo año con Bioquímica me gustó” (varón, 22 años)

“A mí me gustaron los cursos de la carrera” (mujer, 22 años)

"Cuando llevé el curso de Tecnología de los Alimentos me pareció interesante”

(mujer, 22 años)

“A las dos semanas (de empezar las prácticas preprofesionales de Nutrición Aplicada) la Licenciada (personal de la Municipalidad) nos dio la responsabilidad de un proyecto, fue por parejas, yo hice con un interno de (otra universidad). Ellos (los internos de la otra universidad) ya tenían más tiempo (de prácticas), nosotros íbamos a ser su apoyo, pero al momento de las ideas se notó la diferencia en cuanto a planificación, investigación, se vio bastante diferencia, entonces me gustó más” (varón, 21 años).

“Empecé a conocer la labor del nutricionista dentro de muchas áreas, y vi la posibilidad de desarrollarme en varios campos” (mujer 23 años).

"Para contribuir de alguna manera con el bienestar y mejora en la salud del individuo" (mujer 23 años)

"Es importante la influencia que pueden tener nuestras acciones en la vida de la gente" (varón, 22 años)

"Me gusta trabajar directamente con la gente” (varón, 22 años)

"Es más rescatable trabajar cara a cara con la comunidad, cara a cara con la pobreza” (varón, 23 años)
Se muestra algunos comentarios típicos en la Tabla 2. Hubo un participante que ya tenía una visión de lo que podría lograr al estudiar la carrera "Porque hay posibilidad de hacer empresa" (varón, 23 años).

Continuaron sus estudios por desarrollo consecutivo de cursos motivadores, entre ellos los más mencionados fueron ICAN y Nutrición Humana; y por las expectativas de su desempeño en el campo, que fueron satisfechas principalmente durante el décimo semestre.

Entre las razones para concluir la carrera, estuvieron que, tiene diferentes campos de acción (73\%); además, algunos también respondían por la posibilidad de contribuir a mejorar la situación de salud (60\%) y porque les gustaba trabajar directamente con la población (26\%).

Sobre su preparación, en general opinaron favorablemente. Pero recalcaron: "Nos ha faltado que nos orienten más en nutrición deportiva. Más nos enseñan nutrición clínica y comunitaria" (varón, 23 años). También, percibieron que requieren más énfasis y práctica en el área de servicios de alimentación, y para un mejor desempeño en esa área indicaron "Falta un curso de liderazgo (todos lo confirmaron). Los trabajadores (de servicios de alimentación) son mayores y a una la ven joven" (mujer, 22 años). Sugirieron que debían tener prácticas de campo desde el primer semestre, tal como lo hacen otras carreras. 
El $83 \%$ esperaba que la carrera le proporcione éxitos laborales y que le permitiera tener adecuados ingresos económicos; el resto lo dudaba y eran precisamente los que manifestaron que requerían mayor capacitación académica y práctica, mientras que los anteriores manifestaron sentirse aptos para desempeñarse adecuadamente en el área de su preferencia. La mayoría manifestó su deseo de desempeñarse en el área de nutrición pública $(70 \%)$, probablemente porque las experiencias que adquirieron durante sus prácticas preprofesionales de nutrición aplicada, durante el décimo semestre de estudios, fue muy motivadora: "Es el campo que más me agrada (nutrición pública), brinda una visión más global de la realidad nacional, pues se trata a la población en su conjunto y no a paciente por paciente (...); lo más importante es la labor de prevención y promoción de la salud. Es ahí donde está el mayor potencial de los profesionales de $\mathrm{Nu}-$ trición, como entes promotores (de la salud)" (mujer, 23 años), "Definitivamente irme a provincia en el décimo fue la mejor decisión que pude tomar (...); el décimo fue increíble. El contacto con la gente del campo, compartir su vida, sus costumbres, interactuar con otros profesionales, es totalmente diferente, es algo más profundo de lo que nos pueden decir los libros o los profesores de la Escuela. Así se esfuercen por ser lo más didácticos posible. Aprendí que la vida está mucho más allá de mi casa, de los libros, de la Facultad, de la Escuela, pero sobre todo me dí cuenta de algo: hay mucho por hacer" (varón, 22 años).

E1 $30 \%$ de los participantes quisiera desempeñarse en nutrición clínica, mientras que un menor porcentaje del total, preferiría administración de servicios de alimentación, nutrición deportiva y docencia.

Todos esperaban seguir estudiando ya sea una maestría o un diplomado en nutrición pública, nutrición deportiva, administración de empresas, marketing, nutrición pediátrica, nutrición clínica y en tecnología alimentaria; la mayoría esperaba que fuera en Lima, mientras que hay algunos que querían estudiar en Brasil o Chile, u otro país que brindara la especialización de su preferencia.

Los participantes, excepto uno, recomendarían a sus amigos y familiares que estudien Nutrición. El $60 \%$ de los participantes tuvo una experiencia laboral temporal en el campo de la carrera antes de los cinco meses de egresado.

\section{DISCUSIÓN}

Las características socioeconómicas de los egresados habría facilitado la continuidad y culminación de la carrera. La mayoría procedía de colegios privados, todos eran solteros y jóvenes, sin carga familiar y por lo menos la mitad tenía padres y/o madres profesionales. Esto concuerda con las características sociales que tienen los estudiantes argentinos que acceden a las universidades y continúan en ella $\left({ }^{13}\right)$.

Los participantes del estudio ingresaron a la carrera profesional de Nutrición con escaso conocimiento del perfil del nutricionista. Sin embargo, al transcurrir los años de estudios, se afianzó la seguridad de culminarla, por encontrarla atractiva, debido a la variedad de áreas de desempeño laboral y la posibilidad de desarrollo personal y al adquirir sensibilidad social en el campo de la salud: "Sinceramente quisiera escribir, publicar libros (sobre nutrición), hacer investigación en nutrición pública, pertenecer a la FAO" (varón, 22 años).

Las principales razones para estudiar una carrera difieren entre algunos grupos de estudiantes y egresados; los de periodismo, para desarrollar su potencial y por el amor que experimentan hacia el arte de escribir ${ }^{(3)}$, los de enfermería lo hacen para cuidar las personas enfermas $\left({ }^{5}\right)$, los de medicina 
familiar para cuidar de la sociedad y para variar el campo de la práctica $\left({ }^{9}\right)$, y para los estudiantes de nutrición, porque tienen diferentes campos de acción laboral.

Algunos participantes que ingresaron con la idea de trasladarse a otra carrera, desistieron de ello conforme fueron conociendo en qué consistía Nutrición a través de cursos motivadores, como el de Introducción a la Ciencia de la Alimentación y Nutrición, que fue uno de los que se implementó en la actual currícula para el primer semestre, y que estaría cumpliendo su propósito, el de dar a conocer en forma general la carrera y sus diferentes campos de acción al ingresante.

A la mayoría les interesó las prácticas preprofesionales realizadas en la comunidad más que las hospitalarias y de servicios de alimentación. Les atrajo la labor de prevención y promoción de la salud y el tener una relación directa con diferentes grupos de la comunidad. En otros estudios, también se demuestra que la práctica comunitaria suele ser estimulante y decisiva para la elección del área de desempeño laboral $\left({ }^{14-16}\right)$.

La identificación con profesionales de la carrera contribuye a su elección, incluso con personajes de películas de cine, como Patch Adams (personaje médico), para los alumnos brasileños de medicina $\left.{ }^{8}\right)$. Los de Nutrición también se identificaron con nutricionistas exitosos, principalmente con los que destacan en las áreas de nutrición pública y de clínica, lo que probablemente los ha animado a aspirar a desempeñarse en esas áreas. También, se identificaron con algunos profesionales que se desempeñan en docencia, pero a pocos les llama la atención su ejercicio.

En ambos sexos, las motivaciones y el interés por la carrera fueron altas. La mayoría de egresados varones manifestó interés en laborar en el área de nutrición públi- ca y algunos se desempeñaron temporalmente en el área antes de los cinco meses de egresados: "Tres hicimos internado en la Municipalidad (de Lima), les gustó, nos han pedido que nos quedemos (en el Municipio), gracias a nuestro trabajo. Estamos encargados de algunos proyectos en planificación” (varón, 22 años).

Los egresados postularon a la carrera por influencia externa, conveniencia para trasladarse a otra carrera y menor puntaje para el ingreso a la Universidad; continuaron sus estudios porque se presentaron cursos motivadores y por las expectativas de desempeño en campo; y, la concluyeron por la diversidad del campo laboral, por su participación en mejora de la situación de salud de la comunidad y por el trabajo directo con la población

Para la EAPN, se presentan varios retos: difundir la carrera, pues se halló que algunos participantes no sabían de ella hasta prácticamente antes de postular; reestructurar la currícula para que contribuya a satisfacer las expectativas de formación académica y personal de los alumnos, otorgando un mayor peso a la práctica; desarrollar las áreas que aún no tienen mayor atractivo, como el de servicios de alimentación y docencia; y seguir orientándola a las necesidades de la población y al avance tecnológico y científico.

\section{REFERENCIAS BIBLIOGRÁFICAS}

1. Weller KE, Smith BA. Reasons pre-service students choose to teach secondary social studies/social science. Journal of Social Studies Research. 1999;23(2):1-10.

2. Ejieh MUC. Student's reasons for entering Nigerian primary teacher education and their career plans. Research in Education. 2005;74:36-46.

3. Wu W. Motives of Chinese students to choose journalism careers. Journalism \& Mass Communication Educator. 2000;55(1):53-65.

4. McCabe R, Nowak M, Mullen S. Nursing careers: what motivated nurses to choose their profession?. Australian Bulletin of Labour. 2005;31(4):386-406. 
5. Ditommaso E, Rheaume A, Woodside R, Gautreau G. Why students choose nursing: a decade ago, most nursing students said that they chose nursing because they wanted to care for people and help others. Are those desires still true today? The Canadian Nurse. 2003;99(5):25-28.

6. Lopez N, Wadenya R, Berthold P. Effective recruitment and retention strategies for underrepresented minority students: perspectives from dental students. Journal of Dental Education. 2003;67(10):1107-12.

7. Lind D, Cendan J. Two decades of student career choice at the University of Florida: increasingly a lifestyle decision. The American Surgeon. 2003;69(1):53-55.

8. Millan LR, Soares R, Rossi E, Neves de Marco OL, Pereira $\mathrm{M}$, Correa P. Whats is behind a student's choice for becoming a doctor?. Clinics. 2005:60(2):143-50.

9. Wright B, Scott I, Woloschuk W, Brenneis F. Career choice of new medical students at three canadian universities: Family Medicine versus Specialty Medicine. Canadian Medical Association Journal. 2004;170(13):1920-4.

10. Rodríguez G, Gil J, García E. Metodología de la investigación cualitativa. 2da. Edición. Ed. Málaga: Aljibe; 1999.

11. Hernández R, Fernández C, Baptista P. Metodología de la investigación. 3ra. Edición. México: Mc Graw Hill; 2003.

12. Tejada Ripalta L. los postulantes e ingresantes a San Marcos. realidad académica, familiar y socioeconómica en el examen de ingreso 2003. Lima: Universidad Nacional Mayor de San Marcos; 2004.
13. García A. Educación superior: acceso, abandono y graduación en la educación superior argentina. Buenos Aires: Sistema de Información de Tendencias en América Latina; 2005.

14. Morrison J, Murray T. Career preferences of medical students: influence of a new four-week attachment in general practice. Br J Gen Pract. 1997;47(416):184-5.

15. Howe A, Ives G. Does Community-based experience alter career preference? New evidence from a prospective longitudinal cohort study of undergraduated medical students. Medical Education. 2001;35(4):391-7.

16. Playford D, Larson A, Wheatland B. Going country: rural student placement factors associated with future rural employment in nursing and allied health. Aust J Rural Health. 2006;14:14-9.

Manuscrito recibido el 19 de julio de 2007 y aceptado para publicación el 28 de agosto de 2007.

\section{Correspondencia:}

Margot Quintana Salinas

Centro de Investigación de Bioquímica y Nutrición

Facultad de Medicina, UNMSM.

Av. Grau 750. Lima 1, Perú.

Correo-e: mquintanas@unmsm.edu.pe 